\title{
Análisis documental de la evaluación educativa bajo el enfoque socio formativo
}

\author{
Rosa Eugenia Villanueva González \\ r_villanuevagsh3@seg-gto.gob.mx \\ https://orcid.org/0000-0003-1592-4153 \\ Secretaría de Educación del Estado de Guanajuato (SEG) \\ Guanajuato, México. \\ Paula Flora Aniceto Vargas \\ paniceto@ipn.mx \\ https://orcid.org/0000-0002-6634-6210 \\ Instituto Politécnico Nacional (IPN) \\ Ciudad de México, México.
}

Recibido: 18/01/2021 Aceptado: 22/10/21

\begin{abstract}
Resumen
El propósito de este artículo fue analizar el concepto de evaluación educativa desde un punto de vista individual y general. El problema que se intenta resolver es la clarificación de la evaluación educativa y su importancia en el proceso educativo para el logro de los objetivos. Se realizó un análisis documental basado en la cartografía conceptual, en donde se buscaron diversas fuentes de información, para posteriormente aplicar los ejes de la cartografía conceptual. El resultado fue esclarecer lo que es la evaluación educativa y sus principales beneficios en el sector educativo. Se puede establecer que aún existe confusión acerca de la evaluación educativa, lo que llega a generar que dicho tema sea irrelevante en muchos aspectos. Es preciso transformar la idea errónea que tienen acerca de que la evaluación es solamente llenar aspectos administrativos, sino que, al aplicarla se puede demostrar de manera real el avance en los objetivos propuestos.
\end{abstract}

Palabras clave: evaluación del docente, evaluación de la educación, evaluación del estudiante, evaluación formativa, método de evaluación.

\section{Análise documental da avaliação educacional no âmbito da abordagem sócio-formativa.}

\section{Resumo}

O objetivo deste artigo era analisar o conceito de avaliação educacional de um ponto de vista individual e geral. O problema a resolver é a clarificação da avaliação educacional e a sua importância no processo educacional para a realização dos objetivos. Foi realizada uma análise documental baseada na cartografia conceptual, na qual foram procuradas várias fontes de informação, a fim de aplicar posteriormente os eixos da cartografia conceptual. O resultado foi esclarecer o que é a avaliação educacional e os seus principais benefícios no sector educacional. Pode estabelecer-se que ainda existe confusão sobre a avaliação educacional, o que a torna irrelevante em muitos aspectos. É necessário transformar o equívoco de que a avaliação se refere 
apenas ao preenchimento de aspectos administrativos, más que, ao aplicá-la, é possível demonstrar de forma real o progresso nos objectivos propostos.

Palavras-chave: avaliação de professores, avaliação educacional, avaliação estudantil, avaliação formativa, método de avaliação.

\title{
Documentary analysis of educational evaluation under the socio-formative approach.
}

\begin{abstract}
The purpose of this article was to analyze the concept of educational evaluation from an individual and general point of view. The problem to be solved is the clarification of the educational evaluation and its importance in the educational process to achieve the objectives. A documentary analysis based on conceptual mapping was carried out, where various sources of information were sought, to subsequently apply the axes of conceptual mapping. The result was to clarify what educational evaluation is and its main benefits in the educational sector. It can be established that there is still confusion about educational evaluation, which leads to the fact that this topic is irrelevant in many aspects. It is necessary to transform the erroneous idea that evaluation is only to fill administrative aspects, but that, by applying it, the progress in the proposed objectives can be demonstrated in a real way.
\end{abstract}

Keywords: teacher evaluation, education evaluation, student evaluation, formative evaluation, evaluation method.

\section{Introducción}

La sociedad siempre ha tenido necesidad de hacer una evaluación acerca de todo lo que se encuentra inmerso en su entorno para darle un sentido a las cosas o situaciones con las que interactúa. En (ALCARAZ, 2015) se considera que la evaluación ha sido un proceso cambiante conforme a las necesidades de la sociedad, que van desde el año 2000 A.C. hasta la actualidad, partiendo de diversas concepciones y características. Hay similitud con la opinión de (ARAGÓN - BORJA, 2015) quien hace referencia que, en la historia de la humanidad, ha sido evidente el interés por la medición de diversos atributos físicos, psicológicos, etc., los cuales pueden evidenciarse desde los filósofos de la antigüedad, en los avances científicos, astronomía, entre otros.

Desde tiempos pasados, la evaluación educativa, ha sido un tema de interés y debate por parte de diversos autores, quienes muestran un punto de vista diverso, de acuerdo con la sociedad en donde están inmersos, así como las necesidades presentadas por esta, (HIDALGO \& MURILLO, 2015) hacen referencia y consideran que la evaluación educativa es una actividad 
de marcado carácter político, quien hace una definición de la evaluación como una actividad meramente técnica, mencionando su ideología y el mundo que desea.

Por su parte, (RAVELA, 2017) hace una crítica a la forma en que se ha transmitido la información sobre la evaluación educativa a través de los años, ya que se ha extendido a gran escala por medio de programas y proyectos nacionales, qué por falta de interés de docentes, así como su mala formación, ha ido dejando poca apropiación del conocimiento por parte de estos, lo cual se transmite en una aplicación poco fiable.

De igual manera, se pueden encontrar diversas definiciones acerca de lo que es evaluación educativa, las cuales van cambiando con la sociedad y de acuerdo con las necesidades presentadas, (ÁLVAREZ, 2019) considera que a la evaluación pretende enriquecer y mejorar las acciones futuras del alumno, mencionando que brindan información oportuna y puntuales sobre el rendimiento.

La Secretaría de Educación Pública, (2017) cita que la evaluación educativa tiene un sentido formativo con las siguientes finalidades: valorar los aprendizajes de los alumnos, identificar las condiciones que influyen en el aprendizaje y mejorar el proceso docente y otros aspectos del proceso escolar.

Es importante considerar que la evaluación educativa no solo conlleva evaluar a los alumnos, sino también el actuar del docente, ya que al no comprender lo que se está haciendo, sería de gran dificultad realizarlo con los alumnos. (CABERO, 2018) hace referencia que la evaluación del docente tiene como finalidad el mejoramiento de la acción profesional, transformando las prácticas con el propósito formativo de mejorarlas, favoreciendo el aprendizaje del alumno.

Un docente bien preparado, conoce lo que va a trabajar y como lo va a hacer, así como los momentos para llevar a cabo, capacitándose en todo momento para el logro del objetivo. (MARTÍNEZ, 2016) referencia que los docentes se preparan (cursos), de acuerdo con las necesidades detectadas en sus prácticas de enseñanza, repercutiendo directamente en el aprovechamiento escolar de los alumnos.

El propósito que se tiene en este documento es realizar un análisis documental del concepto de evaluación educativa, para que directivos, docentes, padres de familia, así como alumnos, tengan claridad acerca de la importancia que tiene, así como su funcionalidad dentro del sector educativo. (SEP, 2011) establece que la evaluación es un proceso integral y 
sistemático en el cual se puede recopilar información para conocer, analizar, juzgar, obtener evidencias y brindar retroalimentación a los alumnos, así como a padres de familia en su apoyo en el quehacer educativo.

Para realizar el intercambio de opiniones acerca de la evaluación, es de suma importancia el dialogo que se realiza con padres de familia, alumnos y otros docentes, propiciando situaciones reales. (TOBÓN, 2018) hace referencia a la evaluación socioformativa, en donde el apoyo y la retroalimentación constante, hace que los alumnos desarrollen las competencias necesarias para la sociedad del conocimiento.

Se pretende resolver que los docentes tengan mayor claridad acerca del concepto de la evaluación educativa dentro del contexto escolar, ya que forma parte del proceso de conocimiento de desarrollo profesional. (PEÑA, 2016) hace una consideración acerca de que, para algunos docentes, las técnicas de evaluación más adecuadas son las pruebas de conocimientos, ya que podía reducir el tiempo y obtener información del alumno de manera más precisa e individual. Esta práctica sigue siendo común en muchos centros escolares, por lo cual hay poco reconocimiento a la importancia real de la evaluación y la importancia de usar diversas técnicas, así como los alcances que puede llegar a tener para fortalecer la educación de calidad.

Diversas teorías pueden respaldar la importancia de la evaluación, como un factor fundamental para el desarrollo de las personas de forma integral, tomando como referencia distintos tipos de evaluaciones ya sea de manera formal, social o metacognitiva, en donde, en todo momento, se hace referencia de la adquisición del conocimiento.

Durante la edad escolar, los alumnos van adquiriendo de forma constante el conocimiento, el cual se va modificando a través de situaciones reales y significativas para ellos (SALDARRIAGA et al., 2016) cita que el constructivismo concibe el conocimiento como una construcción propia del sujeto que se va produciendo día con día resultado de la interacción de los factores cognitivos y sociales, este proceso se realiza de manera permanente y en cualquier entorno en los que el sujeto interactúa.

Así también, los alumnos llegan a la escuela con conocimientos previos, en donde se ve reflejado diferentes factores familiares, que apoyan al conocimiento del alumno. (ARIAS et al., 2017) alude que la Teoría Psico - genética, nace de la necesidad por dar respuestas a varias interrogantes de carácter epistemológico relacionadas con el origen del conocimiento, mismas 
que no se enmarcan únicamente en descubrir cómo es posible éste, sino que va más allá, tratando de dar respuesta a cuestiones sobre su evolución.

Cada uno de los alumnos, muestra aptitudes diversas, así como formas de aprender, lo cual se ve reflejado al momento de la adquisición del conocimiento y la forma en que interactúa con este, a lo cual los docentes deben de mantenerse atentos en todo momento. (APUD \& APUD 2018) explican que al conocer las inteligencias se puede entender la forma en que se interactúa y en que se aprende. Se toman decisiones y se resuelven problemas dependiendo de las inteligencias que se tienen. Al mismo tiempo, se pueden desarrollar las que se obtuvieron como poco desarrolladas para alcanzar otras inteligencias

Es de suma importancia tener en cuenta la etapa del desarrollo de los alumnos, ya que, a partir de esto, los docentes, pueden determinar formas de trabajo con ellos, así como de evaluación que permita al docente, el desarrollo del conocimiento en las áreas de oportunidad que se presenten. (ORTIZ, 2017) considera que el aprendizaje generativo, se plantea como un proceso y no una acumulación de información, por lo cual se puede afirmar que el conocimiento es el proceso de aprender a partir de información nueva que se obtiene de la experiencia.

Cada autor establece su punto de vista acerca de la adquisición del conocimiento del niño en edades tempranas, por lo cual, con el transcurso del tiempo, estas teorías se han ido fortaleciendo y esclareciendo de acuerdo con su caso. Es muy importante retomar dicha información, ya que entender el proceso de adquisición del conocimiento del alumno nos puede ayudar a entender y a fortalecer cual es el papel del docente en el proceso de evaluación educativa y la forma adecuada de su aplicación, así como su observación, para obtener óptimos resultados (SEP, 2017).

Es muy importante trabajar sobre la falta de claridad acerca del procedimiento y aplicación de la evaluación educativa por parte del docente, supuesto que, los docentes se encuentran trabajando la evaluación educativa de forma constante. (SEP, 2017) maneja diversa información respecto al tema como libros digitales o físicos, revistas, ensayos, etc., en donde se hace referencia acerca de este proceso y de la importancia de la actualización y formación continua de maestro, para poder brindar una educación integral a los alumnos. Muchos docentes desconocen dicha información, por lo cual presentan poca claridad en lo que están trabajando día con día. 
(ESCUDERO, 2019) habla acerca de que la evaluación del profesorado es mucho más que una evaluación de su práctica incide en su formación previa, en su perfeccionamiento y en su desarrollo continuo. La formación y el desempeño de los docentes es uno de los factores principales de la calidad de un sistema educativo.

De acuerdo con lo expuesto, el presente estudio conceptual se enfocó en las siguientes metas: 1- Analizar el concepto de la evaluación educativa de forma histórica y con bibliografía actual. 2- Diferenciar la evaluación educativa de otros conceptos similares. 3- Establecer metodologías de aplicación de la evaluación educativa, esto se hará para fortalecer la adquisición del conocimiento, así como su aplicación de forma cotidiana y real.

\section{Metodología}

\section{Tipo de Estudio}

Se realizó un análisis documental basado en la cartografía conceptual (TOBÓN, 2016) en torno al concepto “evaluación educativa”. El presente análisis documental consistió en hacer investigaciones de los artículos, publicados en revistas y algunos libros y otros documentos relacionados con el tema, seleccionarlos, organizándolos

y analizando la información relevante que permiten dar respuesta acerca de lo que es la evaluación educativa en el contexto escolar. La investigación documental se hizo con apoyo de la herramienta "Google Académico" y Libros en físico.

\section{Técnica de Análisis}

La cartografía conceptual se aplicó como estrategia para hacer el análisis de la información y de los documentos revisados en función de 8 ejes con preguntas centrales, que pretenden aclarar y resolver problemas de contexto relacionado con la evaluación educativa, (TOBÓN, 2015).

A continuación, se describe los 8 ejes de la cartografía conceptual de la evaluación educativa y la forma de aplicación (Cuadro 1). 
Cuadro1 - Ejes de la Cartografía Conceptual

\begin{tabular}{|c|c|c|}
\hline Eje & Pregunta central & Descripción del componente \\
\hline Noción & $\begin{array}{l}\text { ¿Cuál es la etimología, desarrollo } \\
\text { histórico y definición típica de } \\
\text { evaluación educativa?, ¿Cuál es la } \\
\text { definición en función desde la socio } \\
\text { formación? }\end{array}$ & $\begin{array}{l}\text { Se hizo una investigación acerca de la } \\
\text { Etimología, su desarrollo histórico, su } \\
\text { definición desde la socioformación, así como } \\
\text { una definición típica. Todo de forma detallada } \\
\text { y lo más claro posible. }\end{array}$ \\
\hline Categorización & $\begin{array}{l}\text { ¿A qué clase inmediatamente mayor } \\
\text { pertenece el concepto, evaluación } \\
\text { educativa? }\end{array}$ & $\begin{array}{l}\text { Se hizo una investigación acerca de las clases } \\
\text { inmediatamente mayores a las que pertenece } \\
\text { el concepto de evaluación educativa. Se } \\
\text { definieron estas clases y establecieron sus } \\
\text { características. }\end{array}$ \\
\hline Caracterización & $\begin{array}{l}\text { ¿Cuáles son los elementos centrales qué } \\
\text { le dan identidad al concepto de } \\
\text { evaluación educativa? }\end{array}$ & $\begin{array}{l}\text { Se hizo una descripción acerca de cuáles son } \\
\text { las características centrales de la evaluación } \\
\text { educativa }\end{array}$ \\
\hline Diferenciación & $\begin{array}{l}\text { ¿De cuáles otros conceptos cercanos y } \\
\text { que estén en la misma categoría se } \\
\text { diferencia la evaluación educativa? }\end{array}$ & $\begin{array}{l}\text { Se realizó una investigación y descripción } \\
\text { acerca de los conceptos con los cuales se suele } \\
\text { confundir evaluación educativa o que son } \\
\text { similares. Se detalla cada uno de ellos y se } \\
\text { hace la diferenciación. }\end{array}$ \\
\hline División & $\begin{array}{l}\text { ¿En qué subclases o tipos se divide la } \\
\text { evaluación educativa? }\end{array}$ & $\begin{array}{l}\text { Se llevó a cabo una descripción acerca de las } \\
\text { subclases en las cuales se divide la evaluación } \\
\text { educativa y se detalla cada una de ellas. }\end{array}$ \\
\hline Vinculación & $\begin{array}{l}\text { ¿Cómo se relaciona el concepto de } \\
\text { evaluación educativa con determinadas } \\
\text { teorías, procesos sociales - culturales y } \\
\text { referentes epistemológicos que estén } \\
\text { por fuera de la categoría? }\end{array}$ & $\begin{array}{l}\text { Se hizo una descripción de una o varias teorías } \\
\text { que estén fuera de la relación de la evaluación } \\
\text { educativa, pero que aportan información } \\
\text { relacionada para su aplicación. Se describen } \\
\text { estas teorías, así como sus contribuciones }\end{array}$ \\
\hline Metodología & $\begin{array}{l}\text { ¿Cuáles son los elementos } \\
\text { metodológicos mínimos que implica el } \\
\text { abordaje de la evaluación educativa? }\end{array}$ & $\begin{array}{l}\text { Se realizó una descripción del proceso que se } \\
\text { lleva a cabo para la aplicación de la } \\
\text { evaluación educativa. }\end{array}$ \\
\hline Ejemplificación & $\begin{array}{l}\text { ¿Cuál podría ser un ejemplo relevante y } \\
\text { pertinente de la aplicación de la } \\
\text { evaluación educativa? }\end{array}$ & $\begin{array}{l}\text { Se describe un ejemplo real y claro acerca de } \\
\text { la evaluación educativa, siguiendo sus pasos } \\
\text { en situaciones reales. }\end{array}$ \\
\hline
\end{tabular}

Adaptado de "Manual de Cartografía Conceptual", por (Tobón, 2015), CIFE, p. 7 


\section{Fases del Estudio}

El estudio conceptual se llevó a cabo siguiendo las siguientes etapas

Fase 1. Se llevó la búsqueda de diversas fuentes de información que tuvieran relación con la evaluación educativa.

Fase 2. Se seleccionaron fuentes que fueran confiables y relacionadas con la evaluación educativa tomando en cuenta los ejes de la cartografía conceptual

Fase 3. Se llevó a cabo la realización de la cartografía conceptual siguiendo sus 8 ejes, a través de las fuentes de información recabadas.

\section{Criterios de Selección de los Documentos}

Se llevaron a cabo los siguientes criterios para seleccionar los documentos en las bases de datos.

1. Se buscaron artículos y libros mediante las siguientes bases de datos: Google Académico y libros en físico.

2. Se emplearon las siguientes palabras esenciales "evaluación educativa" junto con una o varias de las siguientes palabras complementarias: "evaluación integrada", "evaluación continua", "evaluación diagnóstica", “evaluación formativa", "evaluación sumativa", y "planificación".

3. Se seleccionaron diversos artículos de revistas indexadas y diversas fuentes bibliográficas.

4. Los documentos se seleccionaron dentro de un periodo $2011-2018$

5. Los documentos debían abordar algún elemento de los ocho ejes de la cartografía conceptual.

6. Se encontraron vacíos de información, en abordaje desde el pensamiento complejo y el abordaje desde la sociedad del conocimiento.

\section{Documentos Analizados}

Se llevó a cabo el análisis de diversos documentos seleccionados para el estudio a través de las bases de datos y diversa bibliografía. En la Cuadro 2 se hace un recuento de los artículos, libros y documentos de apoyo para complementar el análisis y darle contexto. 
Cuadro 2-Documentos Analizados en el Estudio de la evaluación educativa

\begin{tabular}{|c|c|c|c|}
\hline Tipo de documento & País & Referencia & Temas claves \\
\hline \multirow{3}{*}{ Articulo } & España & Murillo \& Hidalgo (2015) & $\begin{array}{l}\text {-Evaluación educativa } \\
\text {-Evaluación tradicional } \\
\text {-Evaluación para } \\
\text { transformación }\end{array}$ \\
\hline & México & Tobón (2015) & -Ejes de la cartografía conceptual \\
\hline & México & Zúñiga \& Luna (2017) & $\begin{array}{l}\text {-Instrumento para evaluar la } \\
\text { práctica docente } \\
\text {-Noción de evaluación } \\
\text {-Metodología } \\
\text {-Registro de Observación }\end{array}$ \\
\hline \multirow{3}{*}{ Artículo científico } & Ecuador & $\begin{array}{l}\text { Arrollo, Merino \& Peralvo } \\
\text { (2017) }\end{array}$ & $\begin{array}{l}\text {-Teoría psicogenética } \\
\text {-Aprendizaje } \\
\text {-Estadios de desarrollo } \\
\text {-Educación }\end{array}$ \\
\hline & Ecuador & $\begin{array}{l}\text { Saldarriaga, Bravo } \\
\text { \&Rivadeneira (2016) }\end{array}$ & -Pedagogía contemporánea \\
\hline & México & Martínez (2016) & $\begin{array}{l}\text {-Concepciones sobre la evaluación } \\
\text {-Desempeño docente } \\
\text {-Calidad educativa }\end{array}$ \\
\hline \multirow{3}{*}{$\begin{array}{l}\text { Artículo de revista } \\
\text { indexada }\end{array}$} & España & Salarirche (2015) & $\begin{array}{l}\text {-Historia de la evaluación } \\
\text {-Etapas de la evaluación. }\end{array}$ \\
\hline & Ecuador & Ortiz (2017) & $\begin{array}{l}\text { Aprendizaje generativo } \\
\text {-Metacognición } \\
\text {-Metanoia }\end{array}$ \\
\hline & España & Cabero (2018) & $\begin{array}{l}\text {-Evaluar el desempeño docente } \\
\text { - Objeto de la evaluación } \\
\text {-Proceso de evaluación }\end{array}$ \\
\hline $\begin{array}{l}\text { Artículos de investigación } \\
\text { científica }\end{array}$ & Ecuador & Apud López (2018) & $\begin{array}{l}\text {-Inteligencias múltiples } \\
\text {-Desarrollo cognitivo }\end{array}$ \\
\hline Capítulo de libro & México & Álvarez (2019) & $\begin{array}{l}\text {-Calidad educativa } \\
\text {-Evaluación }\end{array}$ \\
\hline \multirow{4}{*}{ Libro } & Argentina & Cabrera (2016) & $\begin{array}{l}\text {-Pedagogía bancaria } \\
\text {-Evaluación formativa } \\
\text {-Construcción del conocimiento } \\
\text {-Aprendiendo entre pares } \\
\text {-Evaluación educativa }\end{array}$ \\
\hline & México & Ravela \& Loureiro (2017) & $\begin{array}{l}\text {-Evaluación en el aula } \\
\text {-Propuestas de trabajo para } \\
\text { docentes }\end{array}$ \\
\hline & México & SEP (2017) & $\begin{array}{l}\text {-Planes y programas de la } \\
\text { educación básica }\end{array}$ \\
\hline & México & SEP (2012) & 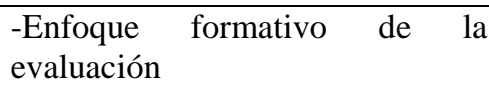 \\
\hline
\end{tabular}


Cuadro 2-Documentos Analizados en el Estudio de la evaluación educativa (continuación)

\begin{tabular}{clll}
\hline Tipo de documento & \multicolumn{1}{c}{ País } & \multicolumn{1}{c}{ Referencia } & \multicolumn{1}{c}{ Temas claves } \\
\hline & México & SEP (2011) & $\begin{array}{l}\text {-Herramientas para la evaluación } \\
\text {-Plan de estudios educación básica }\end{array}$ \\
\cline { 2 - 4 } & México & SEP (2011) & -Guía de la educadora \\
& & & \\
\cline { 2 - 4 } & México & Tobón (2017) & -Tipos de Rúbricas \\
& México & Borja (2015) & $\begin{array}{l}\text {-Evaluación psicológica } \\
\end{array}$ \\
\cline { 2 - 4 } & & & -Historia de la evaluación \\
\hline Revista científica & Venezuela & Peña (2016) & $\begin{array}{l}\text {-Mejora profesional } \\
\text {-Mitos del proceso de evaluación } \\
\end{array}$ \\
& & & -Teoría constructivista de Jean Piaget \\
\hline Revista de investigación & España & Escudero (2019) & $\begin{array}{l}\text {-Modelo educativo } \\
\text {-Ámbitos actitudes } \\
\end{array}$ \\
& & & -Propósitos de la evaluación \\
\hline
\end{tabular}

Fuente: Adaptada de Tobón (2015)

\section{Resultados}

Noción ¿Cuál es la Etimología del concepto evaluación educativa, su desarrollo histórico, su definición actual y desde la socioformación?

\section{Etimología.}

El concepto "Evaluación Educativa", viene de Evaluación del Francés évaluer y Educación del lat. educatio, -ōnis, según la Real Academia Española (2016) lo cual podría traducirse como estimar conocimientos, aptitudes y valores de los alumnos, docentes y demás personas inmersas en el sector educativo.

\section{Desarrollo histórico.}

(ALCARAZ, 2015) hace referencia acerca de que la evaluación ha sido un proceso cambiante conforme a las necesidades de la sociedad, que van desde el 2000 a. de C. hasta la actualidad. (ARAGÓN, 2015) evidencia que, en la historia de la humanidad, ha sido evidente el interés por la medición de diversos atributos físicos, psicológicos, etc. Los cuales pueden evidenciarse desde los filósofos de la antigüedad, en los avances científicos y astrónomos, por lo cual podría decirse que, desde tiempos pasados, la evaluación ha sido un tema de interés para las personas, puesto que este puede proporcionar un "valor" a objetos, personas o situaciones que generen dicho interés, lo cual puede llevar a tomar decisiones sobre las acciones o situaciones a realizar a partir de los resultados. 


\section{Definición actual.}

Durante parte del desarrollo histórico de la evaluación educativa, en el sector escolar, muchos docentes han visto que las pruebas de conocimientos son una técnica de evaluación "más adecuada", ya que pueden reducir el tiempo y obtener información del alumno de manera más precisa e individual. Esto en muchas ocasiones no permite obtener detalles del desarrollo integral de los alumnos, ya que se llega a limitar a un solo aspecto (PEÑA, 2016).

Haciendo un enfoque a lo que es Evaluación Educativa, de manera etimológica, según la (RAE, 2016) Evaluación viene del francés évaluer y Educación del lat. educatio, -ōnis, en donde podría decirse que la evaluación educativa, es estimar los conocimientos, aptitudes y rendimiento de los alumnos, docentes y demás actores que se encuentren inmersos en la comunidad educativa.

De igual manera, para los docentes la evaluación ha llegado a ser un reto constante ya sea aplicado en el salón de clases para con los alumnos, o de forma personal para trabajar en las áreas de oportunidad de manera profesional. (RAVELA, 2017) hace una crítica a dicha conceptualización que se tenía de la evaluación educativa, que en las últimas décadas se ha extendió a gran escala por medio de programas y proyectos nacionales e internacionales, sin embargo, hay poca apropiación por parte del docente acerca de la importancia de la evaluación para sí y para los demás.

Para poder atender estas necesidades que se presentan en la comunidad educativa y que son de manera recurrente, la Secretaría de Educación Pública, comenzó a reformar el concepto que se tiene sobre la evaluación educativa, favoreciendo que tanto alumno, padres de familia, docentes, directivos, supervisores o jefes de sector, puedan tener claridad sobre ello de manera sencilla, abarcando valores, principios, competencias de la vida, entre otros.

(SEP, 2013) explica que la evaluación educativa es un proceso integral y sistemático a través del cual se recopila la información de manera metódica y rigurosa, para conocer, analizar y juzgar el valor de un objeto educativo determinado: los aprendizajes de los alumnos, el desempeño de los docentes, el grado de dominio del currículo y sus características, permitiendo obtener evidencias, elaborar juicios y brindar retroalimentación sobre los logros de aprendizajes.

(ÁLVAREZ, 2019) considera que a la evaluación pretende enriquecer y mejorar las acciones futuras del alumno, mencionando que brindan información oportuna y puntuales sobre el rendimiento. 


\section{Definición desde la socioformación.}

Desde el enfoque socioformativo, considerando la sociedad del conocimiento, se propone la siguiente definición (SEP, 2017) la evaluación educativa tiene un sentido formativo con las siguientes finalidades: valorar los aprendizajes de los alumnos, identificar las condiciones que influyen en el aprendizaje y mejora el proceso docente y otros aspectos del proceso escolar. La evaluación debe servir para tomar decisiones respecto a qué es necesario fortalecer, modificar y evitar.

Esta definición tiene las siguientes ventajas o contribuciones, por lo cual permite tener claridad acerca de lo que se pretende lograr con la evaluación educativa en el sector escolar y hacia dónde se dirige a los alumnos y a los docentes en una sociedad cambiante.

La Figura 1 se describen los ejes esenciales de la evaluación educativa según el PNEE o INEE.

Figura 1 - Ejes esenciales de la evaluación educativa según el PNEE o INEE.

\begin{tabular}{|c|c|c|c|c|c|c|}
\hline $\begin{array}{l}\text { Desarrollo } \\
\text { de } \\
\text { evaluacione } \\
\text { s de los } \\
\text { componente } \\
\text { s, procesos } \\
\text { y resultados } \\
\text { del Sistema } \\
\text { de } \\
\text { Educación } \\
\text { Nacional }\end{array}$ & $\begin{array}{c}\text { Regulaci } \\
\text { ón de los } \\
\text { procesos } \\
\text { de } \\
\text { evaluació } \\
n\end{array}$ & $\begin{array}{c}\text { Desarrollo } \\
\text { del sistema } \\
\text { de } \\
\text { informació } \\
\text { n e } \\
\text { indicadores } \\
\text { clave de } \\
\text { calidad y } \\
\text { equidad } \\
\text { educativa }\end{array}$ & $\begin{array}{c}\text { Difusión } \\
\text { y uso de } \\
\text { los } \\
\text { resultados } \\
\text { de la } \\
\text { evaluació } \\
\text { n }\end{array}$ & $\begin{array}{c}\text { Fortalecimi } \\
\text { ento de las } \\
\text { capacidades } \\
\text { instituciona } \\
\text { les }\end{array}$ & $\begin{array}{l}\text { Coordinaci } \\
\text { ón } \\
\text { instituciona } \\
\text { l entre el } \\
\text { INEE y las } \\
\text { autoridades } \\
\text { educativas }\end{array}$ & $\begin{array}{c}\text { Emisión } \\
\text { y } \\
\text { ejecución } \\
\text { de } \\
\text { directrice } \\
\text { s para la } \\
\text { mejora } \\
\text { educativa }\end{array}$ \\
\hline
\end{tabular}

Fuente: Información tomada de Política Nacional de Evaluación de la Educación (INEE, 201, p 49-90)

Categorización ¿A qué clase inmediatamente mayor pertenece el concepto, evaluación educativa?

El concepto de evaluación educativa se inscribe dentro de la planificación, quien a su vez pertenece a los programas de estudio, los cuales forman parte del perfil de egreso y posteriormente a la educación básica, los cuales se detallan a continuación. 
La planificación, (SEP, 2011) es una forma de organización del trabajo docente que busca ofrecer experiencias significativas a los niños que generen la movilización de sus saberes y la adquisición de otros. Dentro del sector educativo, es una herramienta fundamental para que los docentes puedan plasmar y evidenciar lo que los alumnos necesitan aprender o fortalecer de acuerdo con el diagnóstico previo del maestro.

Posteriormente podemos encontrar lo que son los programas de estudio, en donde se encuentra plasmado lo que los alumnos van a estudiar y que se pretende que aprendan en un tiempo determinado, lo cual ha surgido a partir de las evaluaciones realizadas durante años de investigación para propiciar el desarrollo integral de los alumnos (SEP, et al., 2011).

El perfil de egreso, (SEP, 2011) Define el tipo de alumno que se espera formar en el transcurso de la escolaridad básica. Plantea los rasgos deseables que los estudiantes deberán mostrar al término de la educación básica, de acuerdo con las necesidades cambiantes de la sociedad. Sus razones de ser son:

1- definir el tipo de ciudadano que se espera formar

2- $\quad$ ser un referente común para la definición de los componentes curriculares ser un indicador para valorar la eficiencia del proceso educativo

Así también encontramos lo que es la educación básica (SEP, 2017) la educación básica y la educación media superior conforman la educación obligatoria. La educación básica abarca la formación escolar de los niños desde los tres a los quince años y se cursa a lo largo de doce grados.

Caracterización ¿Cuáles son los elementos centrales qué le dan identidad al concepto de evaluación educativa?

El concepto evaluación educativa, desde el punto de vista de (ARAGÓN, 2015) tiene las siguientes características:

1- Integrada

2- Formativa

3- Continua

4- Recurrente

5- Criterial

6- Decisoria

7- Cooperativa 
A continuación, se explica cada una de estas características:

Integrada: Se encuentra dentro del currículum diseñado para cada nivel educativo, por lo cual la evaluación educativa no va desligada de lo que se pretende favorecer en cada uno de los niveles educativos, lo cual permite poder revalorar lo que se ha planteado y modificar de acuerdo con las nuevas reformas que se actualizan de forma constante respecto la sociedad en la que se encuentra inmersa.

Formativa: Que pueda fortalecer el proceso, así como el resultado de la acción educativa. No solo hay que llevar a cabo la evaluación como requisito dentro del sector educativo, sino, este debe de permitir fortalecer los procesos que se lleven a cabo durante el periodo establecido, así como los resultados que se pretenden alcanzar.

Continua: Se debe de llevar a cabo durante todo el proceso en el ciclo escolar, ya que no puede aparecer de forma aislada en algunos periodos de este ciclo, puesto que al llevarla a cabo de forma continua brinda mejores resultados ante las problemáticas que se presentan dentro del sector educativo y permite tener resultados reales para poder mejorar de forma progresiva.

Recurrente: Qué se lleve a cabo de manera permanente y continua durante todo el proceso. Como se mencionó anteriormente, no se puede llevar a cabo esta evaluación educativa de forma esporádica, ya que los resultados no serían reales y por consecuente no permitirían tomar decisiones acertadas para la mejora continua de los aprendizajes de los alumnos.

Criterial: Referida a los criterios establecidos para todos los alumnos. Mencionando que los criterios deben de ser acordes a la información que se pretende recopilar de cada uno de los estudiantes, guiando en todo momento el logro de los objetivos.

Decisoria: Que permita tomar decisiones con respecto a los resultados arrojados. La evaluación educativa debe de cumplir con la finalidad de propiciar información que sea verídica y real para poder determinar el logro de los objetivos y a partir de ellos, poder tomar decisiones en cuanto a los logros obtenidos, así como las áreas de oportunidad que aún se deben de trabajar.

Cooperativa: Todos los involucrados deben de participar activamente. No únicamente el docente debe de llevar a cabo le evaluación educativa, sino que todos los integrantes de la comunidad escolar deben de formar parte de dicho proceso, para que los resultados sean acordes a la realidad escolar y a los propósitos planteados, Aragón, et al., (2015). 
Diferenciación ¿De cuáles otros conceptos cercanos y que estén en la misma categoría se diferencia la evaluación educativa?

El concepto de evaluación educativa difiere de los siguientes conceptos con los cuales a veces se llega a confundir. A continuación, se menciona cada uno de ellos y se explican las diferencias entre estos, según la (SEP, 2013):

1. Medición

2. Estimación

3. Calificación

4. Acreditación

A continuación, se hace una explicación de cada uno de dichos conceptos:

Medición: Se define como la asignación de un valor numérico a conocimientos, habilidades, valores o actitudes, logrados por los alumnos durante un periodo de corte. Este concepto se diferencia de la evaluación educativa ya que, aunque pueden guardar similitudes, la realidad es que la medición asigna un valor numérico para reportar lo que se aprendió en un momento determinado, mientras que la evaluación educativa permite llevar a cabo un seguimiento continuo acerca del desarrollo del alumno en las etapas del ciclo escolar.

Estimación: Estimar es la acción concreta de emitir un juicio de lo que ha aprendido un alumno, con base en evidencias cualitativas y cuantitativas, cuando sea el caso. Una forma de estimación en el ámbito escolar es la calificación. Respecto a la estimación, podemos mencionar que la evaluación educativa no solo permite determinar en un cierto momento los resultados de los alumnos, sino que propicia que se lleve a cabo la valoración de forma continua y apegada a los cambios reales que se van presentando durante el transcurso del tiempo, tomando en cuenta los factores que le rodean, sin encasillarlos únicamente en un aspecto.

Acreditación: Consiste en toma la decisión pertinente de si un alumno acceda al grado escolar siguiente en función de las evidencias cualitativas y cuantitativas que se tienen del logro de los aprendizajes esperados. Haciendo una comparativa con la evaluación educativa, esta se enfoca en seguir de cerca el desarrollo integral del alumno, independientemente del grado escolar que este cursando.

Calificación: Se refiere solo a la expresión cualitativa (destacado, satisfactorio, suficiente) del nivel de desempeño o cuantitativa $(10,9,8,7 \ldots)$. La evaluación educativa tiene como finalidad propiciar información acerca del desarrollo del alumno en todo el periodo 
escolar, de forma cualitativa, así como cuantitativa, brindado diversas opciones de actuar ante los resultados por parte del docente, así como del alumnado

Clasificación ¿En qué subclases o tipos se divide la evaluación educativa?

El concepto de evaluación educativa se puede clasificar en función a la información que se espera recopilar, así como los momentos en que se lleva a cabo y las personas que la realizan (SEP, 2013) considerando esto, se tienen las siguientes clasificaciones de evaluación educativa.

1. Evaluación diagnostica

2. Evaluación formativa

3. Evaluación sumativa o final

4. Autoevaluación

5. Coevaluación

6. Heteroevaluación

De las cuales se hace una descripción a continuación

Evaluación diagnostica: Se realiza de manera previa al desarrollo de un proceso educativo. Su objetivo es establecer una línea base de aprendizajes comunes para diseñar las estrategias de intervención docente. Se realiza al inicio del ciclo escolar, en una situación o secuencia didáctica. A partir de esta evaluación diagnostica, se puede conocer en donde se encuentran los alumnos y cuáles serán las acciones que se llevarán a cabo para el logro de los objetivos propuestos.

Evaluación Formativa: Se realiza para valorar el avance en los aprendizajes y mejora la enseñanza y el aprendizaje. Su función es mejorar una intervención en un momento determinado. Este tipo de evaluación permite valorar de manera constante el desarrollo de los alumnos, así como de los objetivos establecidos, permitiendo de esta forma poder continuar con las elecciones de las acciones correspondientes para el avance de forma integral.

Evaluación sumativa o final: Promueve que se obtenga un juicio global del grado de avance de los aprendizajes esperados de cada alumno. Se basa en la recolección de información acerca de los resultados de los alumnos. A partir de esta evaluación, se puede determinar el nivel de dominio de los aprendizajes por parte de los alumnos, así como el logro de los objetivos y permite reflexionar y reestructurar el actuar docente.

Autoevaluación: Es la evaluación que realiza el propio alumno de sus producciones y proceso de aprendizaje. Así también puede llevarse a cabo por parte del docente como parte de 
la metacognición. La autoevaluación permite que las personas que lo llevan a cabo logren conocer en donde están y cuál es el rumbo que deben de seguir.

Coevaluación: Es la evaluación que realiza el propio alumno con sus compañeros. En este aspecto, la coevaluación permite al alumno conocer el punto de vista, acerca de cierta información, de otras personas, por lo cual llega a enriquecer y a apropiarse de nuevos conocimientos, así como retroalimentar a sus compañeros, lo cual se convierte en un aprendizaje significativo.

Heteroevaluación: Es la evaluación que el docente realiza de las producciones de sus alumnos. Dicha evaluación permite evidenciar al docente cual es el trabajo que han realizado los alumnos y su nivel de dominio con respecto a los aprendizajes, para poder tomar decisiones respecto a las nuevas acciones que se llevaran a cabo para el logro de dichos objetivos.

El tipo de evaluación que se lleve a cabo dependerá en medida acerca de la información que se quiera recopilar y el tiempo en que esta se lleve a cabo.

Vinculación ¿Cómo se relaciona el concepto de evaluación educativa con determinadas teorías, procesos sociales - culturales y referentes epistemológicos que estén por fuera de la categoría?

El concepto de evaluación educativa se puede vincular con: 1) Constructivismos, 2) Teoría Psico - genética, 3) Inteligencias múltiples y 4) Teoría del procesamiento de la información. A continuación, se explica cada uno de estos procesos de vinculación y las contribuciones que hacen al concepto objeto del presente estudio:

Constructivismo: (SALDARRIAGA et al., 2016) concibe el conocimiento como una construcción propia del sujeto que se va produciendo día con día resultado de la interacción de los factores cognitivos y sociales, este proceso se realiza de manera permanente y en cualquier entorno en los que el sujeto interactúa. Para poder llegar a esta construcción del conocimiento, el alumno en su caso debe de llevar a cabo una autoevaluación de manera constante, en donde la reflexión debe de ser guía para el desarrollo integral, por consecuente, la evaluación esta de forma presente de forma recurrente. Mantiene así también las siguientes características:

1. Concibe al ser humano como un ente autogestor que es capaz de procesar la información obtenida del entorno, interpretarla de acuerdo con lo que ya conoce convirtiéndola en un nuevo conocimiento. 
2. El desarrollo cognoscitivo del sujeto parte de formas hereditarias muy elementales, para ser construido por él mediante un proceso psicogenético.

3. La construcción de los esquemas mentales es elaborada a partir de los esquemas de la niñez, en un proceso de reconstrucción constante.

Teoría Psico-genética: (ARROLLO et al., 2017) nace de la necesidad por dar respuestas a varias interrogantes de carácter epistemológico relacionadas con el origen del conocimiento, mismas que no se enmarcan únicamente en descubrir cómo es posible éste, sino que va más allá, tratando de dar respuesta a cuestiones sobre su evolución. La teoría Psico-genética, tiene como finalidad responder cuestionamientos del origen del conocimiento, al cual podemos acceder a través de una evaluación permanente, permitiendo la reflexión sistemática para poder obtener las respuestas a las interrogantes.

Basa su fundamentación en tres estadios que se presentan en el desarrollo cognitivo:

a) sensorio motor

b) inteligencia representativa o conceptual

c) de operaciones formales.

Inteligencias múltiples: (APUD \& APUD, 2018) al conocer las inteligencias se puede entender la forma en que se interactúa y en que se aprende. Se toman decisiones y se resuelven problemas dependiendo de las inteligencias que tenemos. Al mismo tiempo, se pueden desarrollar las que se obtuvieron como poco desarrolladas para alcanzar otras inteligencias. Para poder conocer dichas inteligencias, la evaluación educativa, forma parte fundamental para poder llevar a cabo la recopilación de datos que permitan evidenciar cual es la inteligencia dominante en cada persona, así como las acciones que se podrían desarrollar para alcanzar otras poco desarrolladas.

A continuación, se mencionan las ocho inteligencias múltiples y sus características:

1. Verbal / Linguiística: Es la capacidad de emplear de manera eficaz las palabras, manipulando la estructura o sintaxis del lenguaje, la fonética, la semántica, y sus dimensiones prácticas.

2. Lógica / Matemática: Es la capacidad de manejar números, relaciones y patrones lógicos de manera eficaz, así como otras funciones y abstracciones de este tipo 
3. Visual / Espacial: Es la habilidad de apreciar con certeza la imagen visual y espacial, de representarse gráficamente las ideas, y de sensibilizar el color, la línea, la forma, la figura, el espacio y sus interrelaciones.

4. Musical / Rítmica: Es la capacidad de percibir, distinguir, transformar y expresar el ritmo, timbre y tono de los sonidos musicales.

5. Corporal / Kinestésica: Es la habilidad para usar el propio cuerpo para expresar ideas y sentimientos, y sus particularidades de coordinación, equilibrio, destreza, fuerza, flexibilidad y velocidad, así como propioceptivas y táctiles.

6. Interpersonal: Es la posibilidad de distinguir y percibir los estados emocionales y signos interpersonales de los demás, y responder de manera efectiva a dichas acciones de forma práctica.

7. Intrapersonal: Es la habilidad de auto introspección, y de actuar consecuentemente sobre la base de este conocimiento, de tener una autoimagen acertada, y capacidad de autodisciplina, comprensión y amor propio.

8. Naturalista: Es la capacidad de distinguir, clasificar y utilizar elementos del medio ambiente, objetos, animales o plantas. Tanto del ambiente urbano como suburbano o rural. Incluye las habilidades de observación, experimentación, reflexión y cuestionamiento de nuestro entorno

Teoría del aprendizaje generativo: (ORTIZ, 2017) considera que el aprendizaje generativo, se plantea como un proceso y no una acumulación de información, por lo cual se puede afirmar que el conocimiento es el proceso de aprender a partir de información nueva que se obtiene de la experiencia. El niño debe de hacer un esfuerzo mental para aprender y que nada es pasivo en este proceso. Para poder llegar a la metacognición, es importante llevar a cabo la autoevaluación, inmersa en la evaluación educativa, la cual brinda información acerca de en donde se encuentra una persona (lo que es capaz de hacer) y cuáles serían las nuevas acciones que podría llevar a cabo para llegar a una zona de desarrollo potencial.

Metodología de Aplicación ¿Cuáles son los elementos metodológicos mínimos que implica el abordaje de la evaluación educativa?

Con base en la revisión documental (SEP, 2013) los ejes metodológicos mínimos para aplicar el tema o el concepto son: 1) Identificar en donde están los alumnos y hasta donde pueden 
llegar, 2) Tener claridad en lo que se enseña (aprendizajes esperados), 3) Fortalecer y comunicar los criterios de evaluación, 4) Retroalimentar a los alumnos, 5) Supervisar el propio desempeño. A continuación, se explican cada uno de estos elementos metodológicos.

1. Identificar en donde están los alumnos y hasta donde pueden llegar: Se puede aplicar una evaluación diagnostica formal y utilizar los resultados para decidir que saben los alumnos y que requieren de mayor trabajo. Es de suma importancia conocer en donde se encuentran los alumnos o la escuela para poder partir de las necesidades presentadas y establecer nuevos objetivos y formas de llegar a este.

2. Tener claridad en lo que se enseña: Los aprendizajes definen lo que se espera de cada alumno, en términos de saber, saber hacer y saber ser. A partir del diagnóstico realizado, se puede tener claridad acerca de cuáles son las áreas de oportunidad a trabajar y cuáles serán las acciones de intervención por parte del docente

3. Fortalecer y comunicar los criterios de evaluación: Compartir con los alumnos los criterios con los que se evaluara, comunicar los propósitos de lo que se espera que logren al final. Es muy importante que los alumnos conozcan que es lo que se pretenden que ellos aprendan y la forma que serán evaluados, puesto que les permitirá tener una visión amplia de cómo podría ser su actuar y las soluciones que podría encontrar ante una dificultad.

4. Retroalimentar a los alumnos: La retroalimentación facilita el aprendizaje. Se debe de usar un ambiente de confianza y desarrollar normas que propicien una crítica constructiva. Así también se debe de llevar a los alumnos a la reflexión propia (autoevaluación) para que logren la adquisición del conocimiento.

5. Supervisar el propio desempeño: Buscar que los alumnos puedan reflexionar sobre lo aprendido o las áreas de oportunidad a trabajar. A partir de que los alumnos reflexionen sobre su propio conocimiento, podrán entonces apropiarse de él y llevarlo a la práctica en su contexto social inmediato.

Ejemplificación ¿Cuál podría ser un ejemplo relevante y pertinente de la aplicación de la evaluación educativa?

De acuerdo con los ejes definidos en la metodología, a continuación, se presenta un ejemplo de evaluación educativa. Ocurrió en el Jardín de Niños “Carmen Serdán”, en la 
comunidad de Los Lorenzos, Guanajuato. A continuación, se ilustra la puesta en práctica de los diferentes ejes:

Contexto: El Jardín de Niños “Carmen Serdán”, se encuentra ubicado en la comunidad de los Lorenzos, Guanajuato. Cuenta con cinco grupos, uno de $1^{\circ}$, dos de $2^{\circ} \mathrm{y}$ dos de $3^{\circ}$, teniendo un total de matrícula de 103 alumnos.

Problema: Durante el ciclo escolar anterior, se trabajaron diversas actividades que pretendían favorecer las necesidades de la escuela y de los alumnos en general, sin embargo, siguen existiendo áreas de oportunidad en cuestión al desarrollo de los aprendizajes de los alumnos de manera general.

Necesidad (es): La necesidad que se presenta, es la continuación del trabajo de los campos de formación de lenguaje Comunicación y Pensamiento Matemático, así como el área de desarrollo de Educación Socioemocional.

Meta (s): Lograr que el $95 \%$ de los alumnos adquieran y fortalezcan sus habilidades básicas de lenguaje y comunicación, pensamiento matemático y educación socioemocional, para que lo puedan aplicar en situaciones reales de su contexto inmediato.

A continuación, se hace una descripción de la metodología que se llevó a cabo para poder elaborar el Plan Escolar de Mejora Continua.

1. Identificar en donde están los alumnos y hasta donde pueden llegar: A inicio de ciclo escolar se elaboró el Plan Escolar de Mejora Continua, en donde se plasmaron los objetivos, metas y acciones a trabajar durante el nuevo ciclo, a partir de un diagnóstico elaborado previamente sobre áreas de oportunidad y necesidades de los alumnos con respecto a los campos de formación y áreas de desarrollo de Lenguaje y Comunicación, Pensamiento Matemático y Educación Socioemocional.

2. Tener claridad en lo que se enseña: A partir de las necesidades detectadas, se llevó a cabo la elaboración de objetivos, metas y acciones que tienen como finalidad favorecer las necesidades detectadas en el contexto escolar, con respecto al aprendizaje de los alumnos, teniendo en cuenta en todo momento los propósitos de la educación básica.

3. Fortalecer y comunicar los criterios de evaluación: Al finalizar su estructuración, se comunicó a padres de familia, así como a alumnos acerca de las acciones que se realizarían, las fechas programadas para dichas acciones, así como los criterios para llevar a cabo la evaluación permanente. 
4. Retroalimentar a los alumnos: Posteriormente se llevó a cabo las acciones para favorecer las acciones propuestas, en donde, se ha llevado a cabo la evaluación del primer corte, dando se evidencia del avance y del logro obtenido hasta el momento, así como las dificultades que se presentan. Durante estos periodos de seguimiento, así como en la evaluación final, se encamina a los alumnos para que lleven a cabo la autoevaluación (la cual también aplican los docentes) acerca de cuáles son las acciones con las que se deben trabajar de forma constante, los logros obtenidos y las áreas de oportunidad para el próximo ciclo escolar.

La relevancia de este ejemplo es muy importante, puesto que es una realidad de las escuelas de nivel básico, ya que la elaboración del PEMC es una herramienta que permite trabajar las necesidades reales de la escuela, así como de los alumnos. El PEMC contribuye a la aplicación de la evaluación educativa, ya que dicha evaluación permite conocer los avances y áreas a trabajar. Los logros que se presentan es que los padres de familia se integren en el desarrollo de sus hijos y que los mismos alumnos avancen en su desarrollo integral.

Imagen 1 - Diagrama de la Cartografía Conceptual

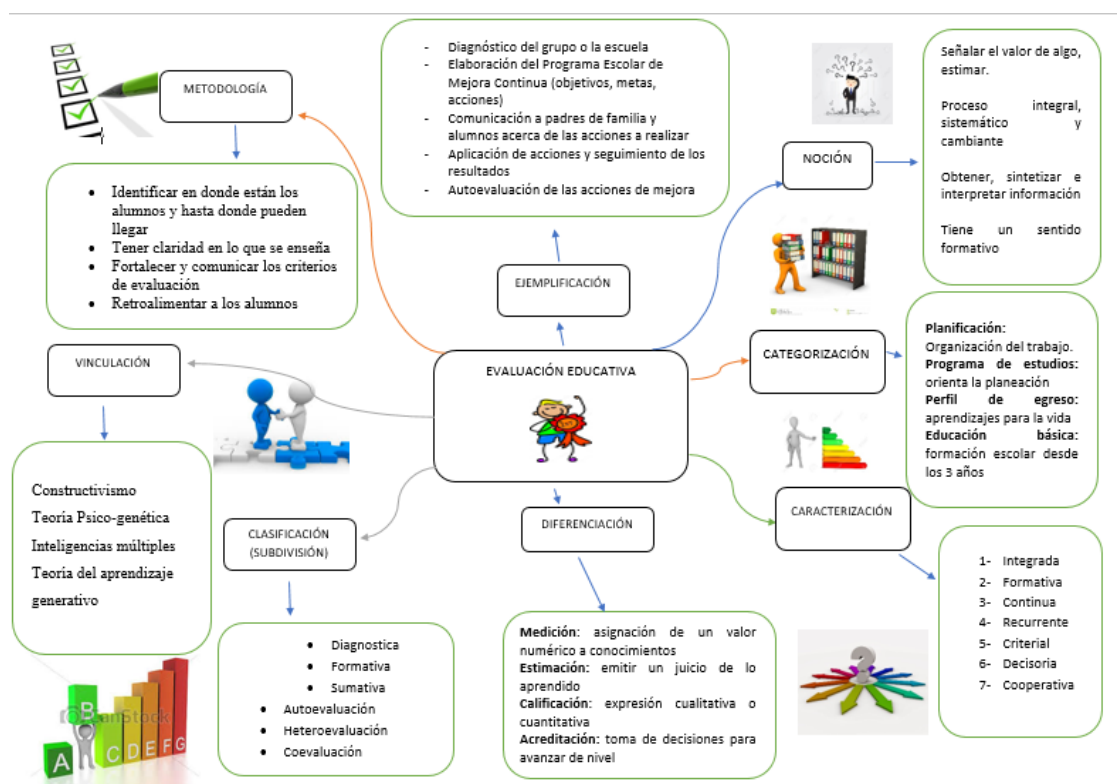

Fuente: Elaboración propia. Tobón, Sergio. (2015). Cartografía conceptual: estrategia para la formación y evaluación de conceptos y teorías.

\section{Discusión}

La evaluación educativa es un proceso fundamental para poder evidenciar los avances en el desarrollo integral de los alumnos. (SEP, 2017) la evaluación tiene un sentido formativo 
con las siguientes finalidades: valorar los aprendizajes de los alumnos, identificar las condiciones que influyen en el aprendizaje y mejora el proceso docente y otros aspectos del proceso escolar. La evaluación debe servir para tomar decisiones respecto a qué es necesario fortalecer, modificar y evitar. En ningún momento la evaluación se debe de ver como un requisito únicamente para llenar documentos, si no, como un espacio de reflexión para el avance del desarrollo integral.

Una segunda conclusión es que, en muchas ocasiones, se sigue confundiendo la evaluación educativa con diversos conceptos, que pueden llegar a generar confusión y una mal aplicación de la evaluación en el sistema educativo, como lo son: medición, estimación, calificación y acreditación (SEP, 2013) debido a que guardan similitud, pero son diferentes entre ellos al momento de adquirir alguna información real de los alumnos.

En tercer lugar, puede concluirse que es importante conocer la metodología de aplicación de la evaluación educativa (SEP, 2013), ya que, al tener claridad en el proceso, es más fácil poder establecer en donde se encuentran los alumnos, lo cual propicia tener claridad acerca de lo que se pretende favorece en ellos, en donde, al comunicar los criterios de evaluación, ya sea a alumnos, padres de familia o personal de la institución, estos puedan apoyar en el desarrollo de forma integral, buscando retroalimentar de manera constante, logrando de esta manera los aprendizajes inicialmente propuestos.

Finalmente, se pudo establecer que la evaluación educativa forma parte fundamental en el actuar diario del docente, ya que permite valorar los aprendizajes de los alumnos, identificando las condiciones diversas y reales que influyen en su aprendizaje y por consecuente mejorar, de forma significativa, el desempeño docente (SEP, 2017).

\section{Referencias}

Albarrán Peña, José Matías, (2016), “Mitos en el proceso de evaluación de los aprendizajes”, Revista Educere, Universidad de los Andes, Venezuela, vol. 20, núm. 65, pp. 73-80, en https://www.redalyc.org/pdf/356/35646429008.pdf, (consulta: 20 noviembre 2019)

Alcaraz Salarirche, Noelia, (2015), “Aproximación Histórica a la Evaluación Educativa: De la generación de la Medición a la Generación Eléctrica", Revista Iberoamericana de Evaluación Educativa. Universidad de Cádiz, vol. 8, núm. 1, p. 12, en https://dialnet.unirioja.es/revista/11882/V/8, (consulta: 15 octubre 2019).

Álvarez Méndez, Juan Manuel. (2019), "La evaluación como actividad crítica de aprendizaje”, ponencia presentada en el "III encuentro de investigación educativa y desarrollo 
pedagógico en el Distrito Capital", Instituto para la investigación educativa y el $\begin{array}{llll}\text { desarrollo } & \text { pedagógico. } & \text { P. } & \text { en }\end{array}$ https://repositorio.idep.edu.co/bitstream/handle/001/2063/III_Encuentro_deInvestigacion_p_227-237.pdf?sequence $=1$, (consulta: 20 octubre 2019)

Apud López, Ramírez Zaira, Apud López, Tammara Ramírez (2018), “Inteligencias múltiples en el trabajo docente y su relación con la teoría del desarrollo cognitivo de Piaget", Revista Killkana Sociales, vol. 22, núm. 2, p 48, DOI: https://doi.org/10.26871/killkana_social.v2i2.299

Aragón Borja, Laura Edna (2015), “Antecedentes históricos de la evaluación psicológica: de la antigüedad del siglo XIX”, en Evaluación psicológica: Historia, fundamentos teórico$\begin{array}{llllll}\text { conceptuales } & y & \text { psicometría, } & 2^{\circ} \text { edición, } \quad \text { p. } 11, \quad \text { en }\end{array}$ https://www.academia.edu/38340689/Evaluaci\%C3\%B3n_psicol\%C3\%B3gica._Histor ia_fundamentos_te\%C3\%B3rico_conceptuales_y_psicometr\%C3\% ADa_ed._2_Laura Edna_Arag\%C3\%B3n_Borja, (consulta: 1 octubre 2019)

Arrollo Arias - Paulina. A., Merino Zurita - Milton. M. \& Peralvo Arequipa - Carmen. R. (2017), "Análisis de la Teoría Psico - genética de jean Piaget: Un aporte a la discusión", Revista científica, dominio de las ciencias. Vol. 3. Número 3, Ecuador. DOI: http://dx.doi.org/10.23857/dom.cien.pocaip.2017.3.3.jun.833-845

Cabero Almenara, Julio., Llorente Cejudo, María del Carmen., \& Morales Lozano, Juan. Antonio. (2018), "Evaluación del desempeño docente en la formación virtual: ideas para la configuración de un modelo. RIED”, Revista Iberoamericana de Educación a Distancia, vol. 21, núm. 1, p. 264, en DOI: http://dx.doi.org/10.5944/ried.21.1.17206

Escudero Escorza, Tomás, (2019), "Evaluación del profesorado como camino directo hacia la mejora de la calidad educativa", Revista de Investigación Educativa, vol. 37, núm.1, 1537, en DOI: http://dx.doi.org/10.6018/rie.37.1.342521

Martínez-Chairez, Guadalupe Iván., Guevara-Araiza, Albertico., \& Valles-Ornelas, María Manuela (2016), "El desempeño docente y la calidad educativa", Revista Ra Ximhai, vol. 12, núm. 6, pp. 123-134, en https://www.redalyc.org/pdf/461/46148194007.pdf, (consulta: 20 enero 2020)

Murillo Torrecilla, F. Javier \& Hidalgo Farran, Nina. (2015). “Dime Como Evalúas y Te Diré Que Sociedad Construyes", Revista Iberoamericana de Evaluación Educativa. Universidad Autónoma de Madrid, vol. 8, núm. 1, pp. 5 - 8, en https://revistas.uam.es/index.php/riee/article/view/2972, (consulta: 10 febrero 2020)

Ortiz, Dorys, (2017), “Aprendizaje generativo, metacognición y metanoia en la formación sistémica”, Revista PUCE, núm. 105, pp. 294, en http://www.revistapuce.edu.ec/index.php/revpuce/article/view/101/157, (consulta: 10 noviembre 2020)

Pedrosa Zúñiga, Luis Horacio. \& Luna, Edna. (2017), "Desarrollo y Validación de un Instrumento para Evaluar la Práctica Docente en Educación Preescolar", Revista Iberoamericana de Evaluación Educativa, Universidad Autónoma de Baja California, vol. 10, núm. $1, \quad$ pp. $109 \quad-\quad 129, \quad$ en https://revistas.uam.es/index.php/riee/article/view/7597/7896, (consulta: 23 enero 2020) 
RAE (2018), Diccionario de la Real Academia Española. Recuperado de: https://dle.rae.es/?id=H8KIdC6, (consulta: 25 septiembre 2019)

Ravela Pedro; Picaroni Beatriz y Loureiro Graciela, (2017), "El elefante invisible”, en ¿Cómo mejorar la evaluación en el aula? Reflexiones y propuestas de trabajo para docentes, Colección Aprendizajes Clave para la educación integral, Primera edición, p. 27-46

Román, Marcela; Murillo F. Javier (2008), “La evaluación del desempeño docente: objeto de disputa y fuente de oportunidades en el campo educativo", Revista Iberoamericana de Evaluación Educativa, vol. 1, núm. 2, p. 5, https://repositorio.uam.es/bitstream/handle/10486/661515/RIEE_1_2_0.pdf?sequence= 1, (consulta: 15 febrero 2020)

Saldarriaga - Zambrano, Pedro J; Bravo - Cedeño, Guadalupe del R; Loor - Rivadeneira, Marlene R, (2016), "La teoría constructivista de Jean Piaget y su significado para la pedagogía contemporánea", Revista científica, Dominio de las Ciencias, vol. 2, núm. 3 Especial, pp. 127 - 137, en https://dialnet.unirioja.es/servlet/articulo? codigo=5802932, (consulta: 20 enero 2020)

Secretaria de Educación Pública (2017) Aprendizajes clave para la educación integral, plan y programa para la educación básica, Ciudad de México, Autor

Secretaria de Educación Pública (2012) El enfoque formativo de la evaluación. Herramientas para la evaluación en educación básica, Dirección general de desarrollo curricular (DGDC), Ciudad de México, Autor

Secretaria de Educación Pública (2011), Plan de estudios. Educación básica, Ciudad de México, Autor

Secretaria de Educación Pública (2011) Programa de estudio 2011, guía para la educadora, Educación Básica Preescolar, Ciudad de México, Autor

Tobón, Sergio, (2018), "Análisis conceptual de la evaluación socioformativa”, en Metodología de la evaluación socioformativa, evaluación socioformativa, Primera edición, pp. 15 32, en https://cife.edu.mx/recursos/wp-content/uploads/2018/08/Libroevaluaci\%C3\%B3n-socioformativa-4.0.pdf\#page=32, (consulta: 3 febrero 2020)

Tobón, Sergio, (2015), Cartografía conceptual, estrategia para la formación y evaluación de conceptos y teorías, México, CIFE

Autores

Rosa Eugenia Villanueva González

Secretaría de Educación del Estado de Guanajuato (SEG), Guanajuato. Graduada como Licenciada en Educación Preescolar, por la Benemérita y Centenaria Escuela Normal Oficial de Guanajuato (BECENOG). En proceso de obtención de grado de Maestría en Docencia y Desarrollo de Competencias, por el Centro Universitario CIFE.

ORCID: https://orcid.org/0000-0003-1592-4153.

E-mail: $\underline{\text { r_villanuevagsh3@seg-gto.gob.mx }}$ 


\section{Paula Flora Aniceto Vargas}

Instituto Politécnico Nacional (IPN), Ciudad de México CDMX.

Nacionalidad: mexicana.

Graduada como Ingeniero Químico Industrial, por el Instituto Politécnico Nacional, en la

Escuela Superior de Ingeniería Química e Industrias Extractivas (ESIQIE), Maestra en Ciencias con especialidad en Sociología Educativa, por el Instituto Ciencias de Irapuato, Maestra en Docencia y Desarrollo de Competencias, por el Instituto CIFE Candidata a Doctora en Talento Humano y Socioformación, por el Instituto CIFE. ORCID: https://orcid.org/0000-0002-6634-6210

E-mail: paniceto@ipn.mx

\section{Como citar el artículo:}

Villanueva González, R. E.; Aniceto Vargas, P. F. Análisis documental de la evaluación educativa bajo el enfoque socio formativo. Revista Paradigma Vol. XLII, Nro. 2, Diciembre de 2021 / $299-324$.

DOI: https://doi.org/10.37618/PARADIGMA.1011-2251.2021.p299-324.id995 